\title{
Editorials
}

\section{The resurgence of tuberculosis and the implications for primary care}

Tuberculosis (TB) is not a disease of the past. The World Health Organization (WHO) estimated that in 2011 there were 8.7 million new cases of TB and 1.4 million people died from the disease. In addition it is estimated that up to one-third of the world's population have latent tuberculosis infection (LTBI). TB is a preventable disease and most forms of tuberculosis are treatable. In recent years the global health community is making progress with concerted and coordinated TB control measures, which have achieved the Millennium Development Goal of halting and reversing the global TB epidemic by 2015.

\section{THE SIZE OF THE UK TUBERCULOSIS PROBLEM}

Closer to home, tuberculosis has been increasing in the UK over the last 30 years, despite significant efforts. TB rates in the UK are now among the highest in Western countries. In 2011 the total number of reported TB cases in the UK was just below 9000, a rate of 14.4 cases per 100000 of the population. ${ }^{1}$ There is significant regional variation and most disease is concentrated in large conurbations. London accounts for the highest proportion of cases (39\%) and the highest rate of disease (44.9 cases per 100000 ) followed by the West Midlands $(11 \%$ and 18.5 cases per 100 000). The majority of TB cases are seen among young adults ( $>60 \%$ cases are aged $15-44$ years) but the majority of TB mortality is seen in older age groups ( $>65$ years). TB is also associated with social risk factors and homeless persons, and those with problem alcohol and illicit drug use, or those with a history of imprisonment are at much greater risk of TB. Nearly three-quarters of TB cases were reported among individuals born outside the UK, many of these were from high TB burden areas including South Asia and sub-Saharan Africa. ${ }^{1}$ The global HIV epidemic also has an influence on UK TB rates and in 2003 8.3\% of TB patients in the UK were co-infected with $\mathrm{HIV}^{2}$, but the proportion is higher in London where co-infection rates are estimated at $17-25 \%{ }^{3}$ but the true UK prevalence of co-infection is unknown.

\section{RAISING AWARENESS AND TB SCREENING IN PRIMARY CARE}

Primary care services can and must play a large role in tackling the rising tide of tuberculosis in the UK. Effective TB control will require early diagnosis of active TB cases, improved identification of those with latent TB most at risk for reactivated disease, and continuing BCG vaccination according to guidelines. ${ }^{4}$ Primary care services are uniquely placed to tackle TB on these three fronts. As primary care physicians we are used to throwing the diagnostic net far and wide and we must be sure to keep tuberculosis in our differential, particularly those of us that work within inner city populations and with non-UK born groups. It is important to remember that the risk of active TB is also increased among patients with diabetes, chronic renal failure, and those receiving anti-TNF agents for rheumatological disease. We may all recognise the classic picture of prolonged cough, haemoptysis, fever, and weight loss but active tuberculosis disease can present in myriad ways and nearly half of TB cases reported in 2011 were extrapulmonary. For example, in this issue Magnussen et al highlight the importance of osteoarticular tuberculosis and the challenge of making a diagnosis. ${ }^{5}$ A recent UK report found that primary care professionals tended to underestimate the rates of TB within their area ${ }^{6}$ and this is understandable given the significant variation in incidence even within one local authority. There are still considerable delays in the recognition and treatment of TB globally lmost studies report a total diagnostic delay ranging between 60-90 days) with contributions from both patient and healthcare provider delays. ${ }^{7}$ Thus, there is still a need to raise awareness of TB within primary care to help tackle diagnostic delay and its contribution to the TB epidemic.

A recent primary care practitioner survey found that in addition to requiring more information on TB diagnostic tests and clearer referral pathways, GPs also wanted to know more about screening for latent TB. ${ }^{6}$ Most TB occurs in non-UK born individuals after they have been resident in the UK for several years ${ }^{1}$ and is due to reactivation of $L T B I$, thus chest $X$-ray screening for active pulmonary disease at port of arrival will have limited effectiveness in controlling TB in the UK. An educational outreach programme in primary care in East London that used verbal screening followed by tuberculin skin testing (TST) was both feasible and successful in improving the proportion of active and latent TB cases identified and in increasing BCG vaccination rates in this setting. ${ }^{8}$ Screening for $\mathrm{LTBI}$ in a primary care setting using interferon gamma release assays compared against TST has been found to be efficacious and cost-effective. Currently there is variation in the practice of screening for latent TB in primary care organisations and those serving the highest TB burden areas are least likely to perform LTBI screening. ${ }^{9}$ Possibly the best setting for LTBI screening will be in primary care in those areas with the highest TB burden. But any programme of this sort would need national coordination and integration between Public Health England, secondary care, and local commissioning groups.

\section{NO BARRIERS TO ACCESSING PRIMARY CARE}

In addition to raising awareness of tuberculosis among primary care professionals there is an ongoing need to work with communities to increase understanding and decrease the stigma of tuberculosis. Improving access of migrants and other risk groups to primary care services is a key component of this, but access to health care is often poor among these groups. Less than one-third of new migrants screened for TB at their port of entry were registered with a GP up to 2 years later. ${ }^{10}$ This is a conservative estimate of the shortfall in new entrant primary care registration, as the study did not include data from illegal migrants who are most at-risk for TB and poor health outcomes. A range of barriers may prevent new migrants from accessing primary care services; these include disincentives to register temporary residents, lack of awareness on eligibility for primary health 


\section{"Success in tackling the rise of TB in the UK will benefit from reminding ourselves of the wider social determinants of health and recognising their importance in informing the new role of GPs in health service design and commissioning.}

care among migrants and GPs, cultural and language barriers, and fears of being reported over immigration status. As GPs we should ensure that our practices offer equitable access to all, and work hard to enable the most vulnerable to access health care without discrimination. Ensuring better access to primary health care supports the individual as well as the wider community through earlier detection of disease and reducing transmission.

\section{THE LINK WITH HIV}

People living with HIV are 20-37 times more likely to develop active TB compared with those without HIV. In addition, HIV is more commonly associated with extrapulmonary and disseminated TB, thus the primary care physician must remain aware of this synergy and be alert for atypical presentations of TB. Since 2008, the British HIV Association and the Health Protection Agency have recommended universal HIV testing in general practice in high incidence areas. Up to one-quarter of persons living with HIV in the UK remain undiagnosed and one-quarter of those newly diagnosed have late stage disease (defined as CD4 counts $\leq 200$ at diagnosis) with a poor prognosis for the individual and increasing risk of onward transmission. ${ }^{11}$ Routinely offering HIV testing in new patient registration health checks is both feasible and acceptable to patients and clinicians. ${ }^{12}$ In 2011 nearly one in five local authorities in England and nine out of 10 in London had an HIV prevalence above the threshold advised for universal testing in primary care. But there has been little in the way of commissioning of HIV testing in the general practice setting.

\section{THE FUTURE}

Throughout history, tuberculosis has often reflected wider social determinants of health. In the UK, TB disproportionately affects individuals from high TB burden countries and hard-to-reach groups with social risk factors, incidence increases with higher levels of deprivation and diagnostic delay is affected by deprivation and ethnicity. ${ }^{13}$ Primary health care has always prioritised equality of access and the redressing of health inequalities through a broad view of health and partnership and integration with social care and community groups. Success in tackling the rise of TB in the UK will benefit from reminding ourselves of the wider social determinants of health and recognising their importance in informing the new role of GPs in health service design and commissioning. Tuberculosis not only tests our diagnostic and clinical skills but reminds us of the pivotal role that primary care plays in protecting the individual and the community from disease.

\section{Louise Pealing,}

London School of Hygiene and Tropical Medicine, Department of Clinical Research, London.

\section{David Moore,}

London School of Hygiene and Tropical Medicine, TB Centre, Department of Clinical Research, London.

\section{Dominik Zenner,}

Centre for Infectious Disease Surveillance and Control, Public Health England, and University College London.

\section{Provenance}

Not commissioned; not externally peer reviewed.

\section{Resources for primary care physicians}

1. RCGP elearning on tuberculosis in primary care. http://www.elearning.rcgp.org.uk/

2. HPA Migrant Health Guide. http://www.hpa.org. uk/MigrantHealthGuide/

3. TB materials on HPA website. http://www.hpa org.uk/Topics/InfectiousDiseases/InfectionsAZ/ Tuberculosis/NationalKnowledgeServiceTB/

DOI: 10.3399/bjgp13X669077

\section{ADDRESS FOR CORRESPONDENCE}

\section{Louise Pealing}

London School of Hygiene and Tropical Medicine, Department of Clinical Research, Keppel Street, London, WC1E 7HT, UK.

E-mail: louise.pealingalshtm.ac.uk

\section{REFERENCES}

1. Health Protection Authority. Tuberculosis in the UK: 2012 report. London: HPA, 2012.

2. Ahmed AB, Abubakar I, Delpech V, et al. The growing impact of HIV infection on the epidemiology of tuberculosis in England and Wales: 1999 2003. Thorax 2007; 62(8): 672-676.

3. Anderson SR, Maguire H, Carless J. Tuberculosis in London: a decade and a half of no decline [corrected]. Thorax 2007; 62(2): 162-167.

4. National Institute for Health and Care Excellence. Clinical diagnosis and management of tuberculosis, and measures for its prevention and control. CG117. London NICE, 2011.

5. Magnussen A, Dinneen A, Ramesh P. Osteoarticular tuberculosis: increasing incidence of a difficult clinical diagnosis. $\mathrm{Br} \mathrm{J}$ Gen Pract 2013; 63: 385-386.

6. Health Protection Authority. Help reverse 20 years of rising Tuberculosis: primary care practitioner survey UK 2010.

London: HPA, 2010. http://www.hpa.org. uk/webw/HPAweb\&HPAwebStandard/ HPAweb_C/1317138449844 laccessed 24 May 2013).

6. Storla DG, Yimer S, Bjune GA. A systematic review of delay in the diagnosis and treatment of tuberculosis. BMC Public Health 2008; 8: 15.

7. Griffiths C, Sturdy P, Brewin P, et al. Educational outreach to promote screening for tuberculosis in primary care: a cluster randomised controlled trial. Lancet 2007; 369(9572): 1528-1534.

8. Pareek M, Abubakar I, White PJ, et al. Tuberculosis screening of migrants to lowburden nations: insights from evaluation of UK practice. Eur Respir J 2011; 37(5): 1175-1182.

9. Stagg HR, Jones J, Bickler G, Abubakar I. Poor uptake of primary healthcare registration among recent entrants to the UK: a retrospective cohort study. BMJ Open 2012; 2(4). pii: e001453

10. Health Protection Agency. HIV in the United Kingdom: 2012 report. London: Health Protection Services, 2012.

11. Prost A, Griffiths CJ, Anderson J, et al. Feasibility and acceptability of offering rapid HIV tests to patients registering with primary care in London (UK): a pilot study. Sex Transm Infect 2009; 85(5): 326-329.

12. French CE, Kruijshaar ME, Jones JA Abubakar I. The influence of socio-economic deprivation on tuberculosis treatment delays in England, 2000-2005. Epidemiol Infect 2009; 137(4): 591-596. 